\title{
Fast Node Overlap Removal
}

\author{
Tim Dwyer ${ }^{1}$, Kim Marriott ${ }^{1}$, and Peter J. Stuckey ${ }^{2}$ \\ 1 School of Comp. Science \& Soft. Eng., Monash University, Australia \\ \{tdwyer, marriott\}@mail.csse.monash.edu.au \\ 2 NICTA Victoria Laboratory \\ Dept. of Comp. Science \& Soft. Eng., University of Melbourne, Australia \\ pjs@cs.mu.oz.au
}

\begin{abstract}
The problem of node overlap removal is to adjust the layout generated by typical graph drawing methods so that nodes of non-zero width and height do not overlap, yet are as close as possible to their original positions. We give an $O(n \log n)$ algorithm for achieving this assuming that the number of nodes overlapping any single node is bounded by some constant. This method has two parts, a constraint generation algorithm which generates a linear number of "separation" constraints and an algorithm for finding a solution to these constraints "close" to the original node placement values. We also extend our constraint solving algorithm to give an active set based algorithm which is guaranteed to find the optimal solution but which has considerably worse theoretical complexity. We compare our method with convex quadratic optimization and force scan approaches and find that it is faster than either, gives results of better quality than force scan methods and similar quality to the quadratic optimisation approach.
\end{abstract}

Keywords: graph layout, constrained optimization, separation constraints.

\section{Introduction}

Graph drawing has been extensively studied over the last twenty years [1]. However, most research has dealt with abstract graph layout in which nodes are treated as points. Unfortunately, this is inadequate in many applications since nodes frequently have labels or icons and a layout for the abstract graph may lead to overlaps when these are added. While a few attempts have been made at designing layout algorithms that consider node size (e.g. 2, 3, 4]), the approaches are specific to certain layout styles and to the best of the authors' knowledge none are perfect in all situations.

For this reason, a number of papers, e.g. [5, 6, 7, 8, 9, 10, have described algorithms for performing layout adjustment in which an initial graph layout is modified so that node overlapping is removed. The underlying assumption is that the initial graph layout is good so that this layout should be preserved when removing the node overlap. Lyons et al. 10] offered a technique based on iteratively moving nodes to the centre of their Voronoi cells until crossings are removed. 
Misue et al. 5] propose several models for a user's "mental map" based on orthogonal ordering, proximity relations and topology and define a simple heuristic Force Scan algorithm (FSA) for node-overlap removal that preserves orthogonal ordering. Hayashi et al. 7] propose a variant algorithm (FSA') that produces more compact drawings while still preserving orthogonal ordering. They also show that this problem is NP-complete. Various other improvements to the FSA method exist and a survey is presented by Li et al. 11. More recently, Marriott et al. 6] investigated a quadratic programming (QP) approach which minimises displacement of nodes while satisfying non-overlap constraints. Their results demonstrate that the technique offers results that are preferable to FSA in a number of respects, but require significantly more processing time. In this paper we address the last issue.

Our contribution consists of two parts: first, we detail a new algorithm for computing the linear constraints to ensure non-overlap in a single dimension. This has worst case complexity $O(n \log n)$ where $n$ is the number of nodes and generates $O(n)$ non-overlap constraints - assuming that the number of nodes overlapping a single node is bounded by some constant $k$. Previous approaches have had quadratic or cubic complexity and as far as we are aware it has not been previously realized that only a linear number of non-overlap constraints are required. Each non-overlap constraint has the form $u+a \leq v$ where $u$ and $v$ are variables and $a \geq 0$ is a constant. Such constraints are called separation constraints. Our second contribution is to give a simple algorithm for solving quadratic programming problems of the form: minimize $\sum_{i=1} v_{i}$. weight $\times\left(v_{i}-v_{i} \text {.des }\right)^{2}$ subject to a conjunction of separation constraints over variables $v_{1}, \ldots, v_{n}$ where $v_{i}$.des is the desired value of variable $v_{i}$ and $v_{i}$.weight $\geq 0$ the relative importance. We show that in practice this algorithm produces optimal solutions to the quadratic program much faster than generic solvers, but also that first part of the algorithm can be run alone to produce near optimal solutions in $O(n \log n)$ time.

\section{Background}

We assume that we are given a graph $G$ with nodes $V=\{1, \ldots, n\}$, a width, $w_{v}$, and height, $h_{v}$, for each node $v \in V 1$ and an initial layout for the graph $G$, in which each node $v \in V$ is placed at $\left(x_{v}^{0}, y_{v}^{0}\right)$ and $u \neq v \Rightarrow\left(x_{u}^{0}, y_{u}^{0}\right) \neq\left(x_{v}^{0}, y_{v}^{0}\right)$.

We are concerned with layout adjustment: we wish to preserve the initial graph layout as much as possible while removing all node label overlapping. A natural heuristic to use for preserving the initial layout is to require that nodes are moved as little as possible. This corresponds to the Proximity Relations mental map model of Misue et al. [5].

Following [6] we define the layout adjustment problem to be the constrained optimization problem: minimize $\phi_{\text {change }}$ subject to $C^{\text {no }}$ where the variables of the layout adjustment problem are the $x$ and $y$ coordinates of each node $v \in V$, $x_{v}$ and $y_{v}$ respectively, and the objective function minimizes node movement

\footnotetext{
${ }^{1}$ Any extra padding required to ensure a minimal separation between nodes is included in $w_{v}$ and $h_{v}$.
} 
$\phi_{\text {change }}=\phi_{x}+\phi_{y}=\sum_{v \in V}\left(x_{v}-x_{v}^{0}\right)^{2}+\left(y_{v}-y_{v}^{0}\right)^{2}$, and the constraints $C^{n o}$ ensure that there is no node overlapping. That is, for all $u, v \in V, u \neq v$ implies

$$
\begin{gathered}
x_{v}-x_{u} \geq \frac{1}{2}\left(w_{v}+w_{u}\right)(v \text { right of } u) \vee x_{u}-x_{v} \geq \frac{1}{2}\left(w_{v}+w_{u}\right)(u \text { right of } v) \\
\vee y_{v}-y_{u} \geq \frac{1}{2}\left(h_{v}+h_{u}\right)(v \text { above } u) \quad \vee y_{u}-y_{v} \geq \frac{1}{2}\left(h_{v}+h_{u}\right)(u \text { above } v)
\end{gathered}
$$

A variant of this problem is when we additionally require that the new layout preserves the orthogonal ordering of nodes in the original graph, i.e., their relative ordering in the $x$ and $y$ directions. This is a heuristic to preserve more of the original graph's structure. Define $C_{x}^{o o}=\bigwedge\left\{x_{v} \geq x_{u} \mid x_{v}^{0} \geq x_{u}^{0}\right\}$ and $C_{y}^{o o}$ equivalently for $y$. The orthogonal ordering problem adds $C_{x}^{o o} \wedge C_{y}^{o o}$ to the constraints to solve.

Our approach to solving the layout adjustment problem is based on [6] where quadratic programming is used to solve a linear approximation of the layout adjustment problem. There are two main ideas behind the quadratic programming approach. The first is to approximate each non-overlap constraint in $C^{\text {no }}$ by one of its disjuncts. The second is to separate treatment of the $x$ and $y$ dimensions, by breaking the optimization function and constraint set into two parts. Separating the problem in this way improves efficiency by reducing the number of constraints considered in each problem and if we solve for the $x$ direction first, it allows us to delay the computation of $C_{y}^{n o}$ to take into account the node overlapping which has been removed by the optimization in the $x$ direction.

\section{Generating Non-overlap Constraints}

We generate the non-overlap constraints in each dimension in $O(|V| \log |V|)$ time using a line-sweep algorithm related to standard rectangle overlap detection methods [12. First, consider the generation of horizontal constraints. We use a vertical sweep through the nodes, keeping a horizontal "scan line" list of open nodes with each node having references to its closest left and right neighbors (or more exactly the neighbors with which it is currently necessary to generate a non-overlap constraint). When the scan line reaches the top of a new node, this is added to the list and its neighbors computed. When the bottom of a node is reached the the separation constraints for the node are generated and the node is removed from the list.

The detailed algorithm is shown on the left of Figure 1. It uses a vertically sorted list of events to guide the movement of the scan_line. An event is a record with three fields, kind which is either open or close respectively indicating whether the top or bottom of the node has been reached, node which is the node name, and posn which is the vertical position at which this happens.

The scan_line stores the currently open nodes. We use a red-black tree to provide $O(\log |V|)$ insert, remove, next_left and next_right operations. The functions new, insert and remove create and update the scan line. The functions next_left(scan_line, $v$ ) and next_right(scan_line, $v$ ) return the closest neighbors to each side of node $v$ in the scan line. 

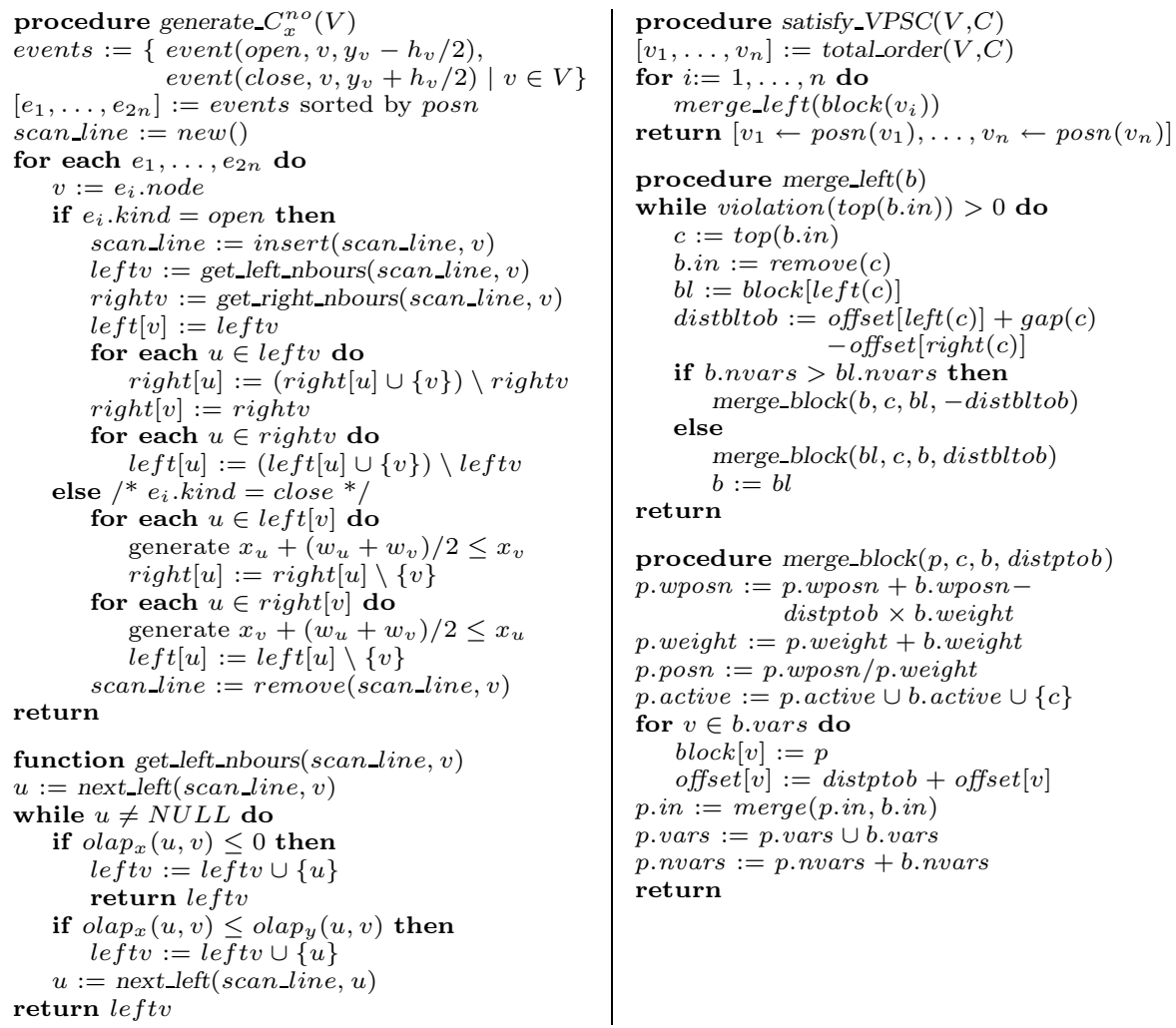

Fig. 1. Algorithm generate_ $C_{x}^{n o}(V)$ to generate horizontal non-overlap constraints between nodes in $V$, and algorithm satisfy_ $V P S C(V, C)$ to satisfy the Variable Placement with Separation Constraints (VPSC) problem

The functions get_left_nbours(scan_line, $v)$ and get_right_nbours $($ scan_line, $v)$ detect the neighbours to each side of node $v$ that require non-overlap constraints. These are heuristics. It seems reasonable to set up a non-overlap constraint with the closest non-overlapping node on each side and a subset of the overlapping nodes. One choice for get_left_nbours is shown in Figure 1] This makes use of the functions $\operatorname{olap}_{x}(u, v)=\left(w_{u}+w_{v}\right) / 2-\left|x_{u}^{0}-x_{v}^{0}\right|$ and $\operatorname{olap}_{y}(u, v)=\left(h_{u}+h_{v}\right) / 2-\left|y_{u}^{0}-y_{v}^{0}\right|$ which respectively measure the horizontal and vertical overlap between nodes $u$ and $v$. The main loop iteratively searches left until the first non-overlapping node to the left is found or else there are no more nodes. Each overlapping node $u$ found on the way is collected in leftv if the horizontal overlap between $u$ and $v$ is less than the vertical overlap. The arrays left and right detail for each open node $v$ the nodes to each side for which non-overlap constraints should be generated. The only subtlety is that redundant constraints are removed, i.e. if there is currently a non-overlap constraint between any $u \in l e f t v$ and $u^{\prime} \in$ rightv then it can be removed since it will be implied by the two new non-overlap constraints between $u$ and $v$ and $v$ and $u^{\prime}$. 
Theorem 1. The procedure generate_C $C_{x}^{\text {no }}(V)$ has worst-case complexity $O(|V|$. $k(\log |V|+k)$ where $k$ is the maximum number of nodes overlapping a single node with appropriate choice of heap data structure. Furthermore, it will generate $O(k \cdot|V|)$ constraints.

Proofs to theorems are provided in the technical report 13. Assuming $k$ is bounded, the worst case complexity is $O(|V| \log |V|)$.

Theorem 2. The procedure generate_ $C_{x}^{n o}(V)$ generates separation constraints $C$ that ensure that if two nodes do not overlap horizontally in the initial layout then they will not overlap in any solution to $C$.

The code for generate_ $C_{y}^{n o}$, the procedure to generate vertical non-overlap constraints is essentially dual to that of generate_ $C_{x}^{n o}$. The only difference is that any remaining overlap must be removed vertically. This means that we need only find the closest node in the analogue of the functions get_left_nbours and get_right_nbours since any other nodes in the scan line will be constrained to be above or below these. This means that the number of left and right neighbours is always 1 or less and gives us the following complexity results:

Theorem 3. The procedure generate_C $C_{y}^{\text {no }}(V)$ has worst-case complexity $O(|V|$. $\log |V|)$. Furthermore, it will generate no more than $2 \cdot|V|$ constraints.

Theorem 4. The procedure generate_ $C_{y}^{n o}(V)$ generates separation constraints $C$ that ensure that no nodes will overlap in any solution to $C$.

\section{Solving Separation Constraints}

Non-overlap constraints $c$ have the form $u+a \leq v$ where $u, v$ are variables and $a \geq 0$ is the minimum gap between them. We use the notation left(c), right $(c)$ and $\operatorname{gap}(c)$ to refer to $u, v$ and $a$ respectively. Such constraints are called separation constraints. We must solve the following constrained optimization problem for each dimension:

Variable placement with separation constraints (VPSC) problem. Given $n$ variables $v_{1}, \ldots, v_{n}$, a weight $v_{i}$.weight $\geq 0$ and a desired value $v_{i}$.des ${ }^{2}$ for each variable and a set of separation constraints $C$ over these variables find an assignment to the variables which minimizes $\sum_{i=1}^{n} v_{i}$.weight $\times\left(v_{i}-v_{i} \text {.des }\right)^{2}$ subject to $C$.

We can treat a set of separation constraints $C$ over variables $V$ as a weighted directed graph with a node for each $v \in V$ and an edge for each $c \in C$ from left $(c)$ to right $(c)$ with length $\operatorname{gap}(c)$. We call this the constraint graph. We define $\operatorname{out}(v)=\{c \in C \mid \operatorname{left}(c)=v\}$ and $\operatorname{in}(v)=\{c \in C \mid \operatorname{right}(c)=v\}$. Note that edges in this graph are not the edges in the original graph.

We restrict attention to VPSC problems in which the constraint graph is acyclic and for which there is at most one edge between any pair of variables.

\footnotetext{
$\overline{{ }^{2} v_{i} . d e s}$ is set to $x_{v i}^{0}$ or $y_{v i}^{0}$ for each dimension, as used in generate_ $C_{\{x \mid y\}}^{n o}$.
} 
It is possible to transform an arbitrary satisfiable VPSC problem into a problem of this form and our generation algorithm will generate constraints with this property. Since the constraint graph is acyclic it imposes a partial order on the variables: we define $u \preceq_{C} v$ iff there is a (directed) path from $u$ to $v$ using the edges in separation constraint set $C$. We will make use of the function total_order $(V, C)$ which returns a total ordering for the variables in $V$, i.e. it returns a list $\left[v_{1}, \ldots, v_{n}\right]$ s.t. for all $j>i, v_{j} \npreceq_{C} v_{i}$.

We first give a fast algorithm for finding a solution to the VPSC algorithm which satisfies the separation constraints and which is "close" to optimal. The algorithm works by merging variables into larger and larger "blocks" of contiguous variables connected by a spanning tree of active constraints, where a constraint $u+a \leq v$ is active if at the current position for $u$ and $v, u+a=v$.

The algorithm is shown in Figure 1. It takes as input a set of separation constraints $C$ and a set of variables $V$. A block $b$ is a record with the following fields: vars, the set of variables in the block; nvars, the size of vars; active, the set of constraints between variables in vars forming the spanning tree of active constraints; in, the set of constraints $\{c \in C \mid \operatorname{right}(c) \in$ b.vars and left $(c) \notin$ b.vars $\}$; out, out-going constraints defined symmetrically to in; posn, the position of the block's "reference point"; wposn, the sum of the weighted desired locations of variables in the block; and weight, the sum of the weights of the variables in the block.

In addition, the algorithm uses two arrays blocks and offset indexed by variables where block $[v]$ gives the block of variable $v$ and offset $[v]$ gives the distance from $v$ to its block's reference point. Using these we define the function $\operatorname{posn}(v)=\operatorname{block}(v)$.posn + offset $[v]$ giving the current position of variable $v$.

The constraints in the field b.in for each block $b$ are stored in a priority queue such that the top constraint in the queue is always the most violated where violation $(c)=\operatorname{left}(c)+g a p(c)-\operatorname{right}(c)$. We use four queue functions: new () which returns a new queue, $\operatorname{add}(q, C)$ which inserts the constraints in the set $C$ into the queue $q$ and returns the result, $\operatorname{top}(q)$ which returns the constraint in $q$ with maximal violation, remove $(q)$ which deletes the top constraint from $q$, and merge $\left(q_{1}, q_{2}\right)$ which returns the queue resulting from merging queues $q_{1}$ and $q_{2}$. The only slight catch is that some of the constraints in b.in may be internal constraints, i.e. constraints which are between variables in the same block. Such internal constraints are removed from the queue when encountered. Another caveat is that when a block is moved violation changes value. However, the ordering induced by violation $(c)$ does not change since all variables in the block will be moved by the same amount and so violation $(c)$ will be changed by the same amount for all non-internal constraints. This consistent ordering allows us to implement the priority queues as pairing heaps [14] with efficient support for the above operations.

The main procedure, satisfy_VPSC, processes the variables from smallest to greatest based on a total order reflecting the constraint graph. At each stage the invariant is that we have found an assignment to $v_{1}, . ., v_{i-1}$ which satisfies the separation constraints. We process vertex $v_{i}$ as follows. First, function block is 


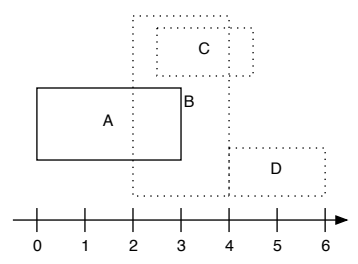

(a)

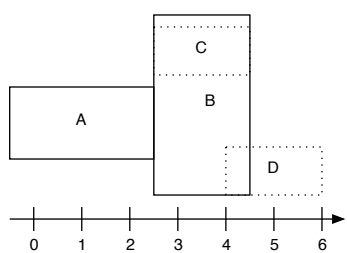

(b)

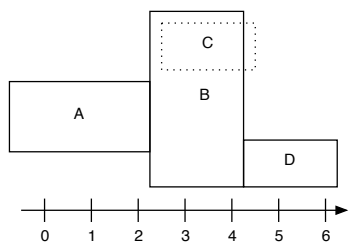

(d)

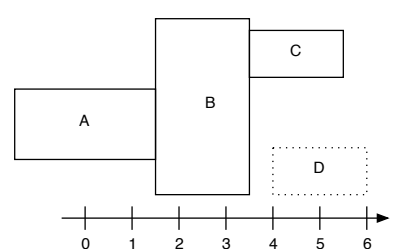

(c)

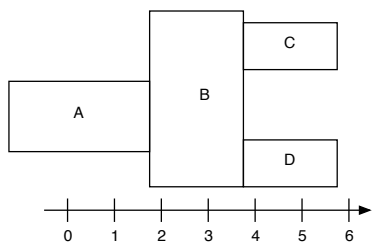

(e)

Fig. 2. Example of (non-optimal) algorithm for VPSC problem giving optimal (c) or non-optimal (e) answer

used to create a block $b$ for each $v_{i}$ setting b.posn $=v_{i}$.des. Some of the "in" constraints may be violated. If so, we find the most violated constraint $c$ and merge the two blocks connected by $c$ using the function merge_block. We repeat this until the block no longer overlaps the preceding block, in which case we have found a solution to $v_{1}, \ldots, v_{i}$.

At each step we set b.posn for each block $b$ to the optimum position, i.e. the weighted average of the desired positions: $\frac{\sum_{i=1}^{k} v_{i} \text {.weight } \times\left(\text { offset }\left[v_{i}\right]-v_{i} . \text { des }\right)}{\sum_{i=1}^{k} v_{i} \text {.weight }}$. By maintaining the fields wposn and weight we are able to efficiently compute the weighted arithmetic mean when merging two blocks.

Example 1. Consider the example of laying out the boxes A,B,C,D shown in Figure2(a) each shown at their desired position 1.5, 3, 3.5, and 5 respectively and assuming the weights on the boxes are 1,1,2 and 2 respectively. The constraints generated by generate_ $C_{x}^{n o}$ are $c_{1} \equiv v_{A}+2.5 \leq v_{B}, c_{2} \equiv v_{B}+2 \leq v_{C}$ and $c_{3} \equiv v_{B}+2 \leq v_{D}$. Assume the algorithm chooses the total order A,B,C,D. First we add block A, it is placed at its desired position as shown in Figure 2(a). Next we consider block $\mathrm{B}$, b.in $=\left\{c_{1}\right\}$ and the violation of this constraint is 1. We retrieve $b l$ as the block containing $A$. and calculate distbltob as 2.5. We now merge block B into the block containing A. The new block position is 1 as shown in Figure 2(b), and $c_{1}$ is added to the active constraints. Next we consider block $\mathrm{C}$, we find it must merge with block AB. The new positions are shown in Figure 2(c). Since there is no violation with the block D, the final position leaves it where it is, i.e. the result is optimal.

Theorem 5. The assignment to the variables $V$ returned by satisfy_VPSC $(V, C)$ satisfies the separation constraints $C$. 

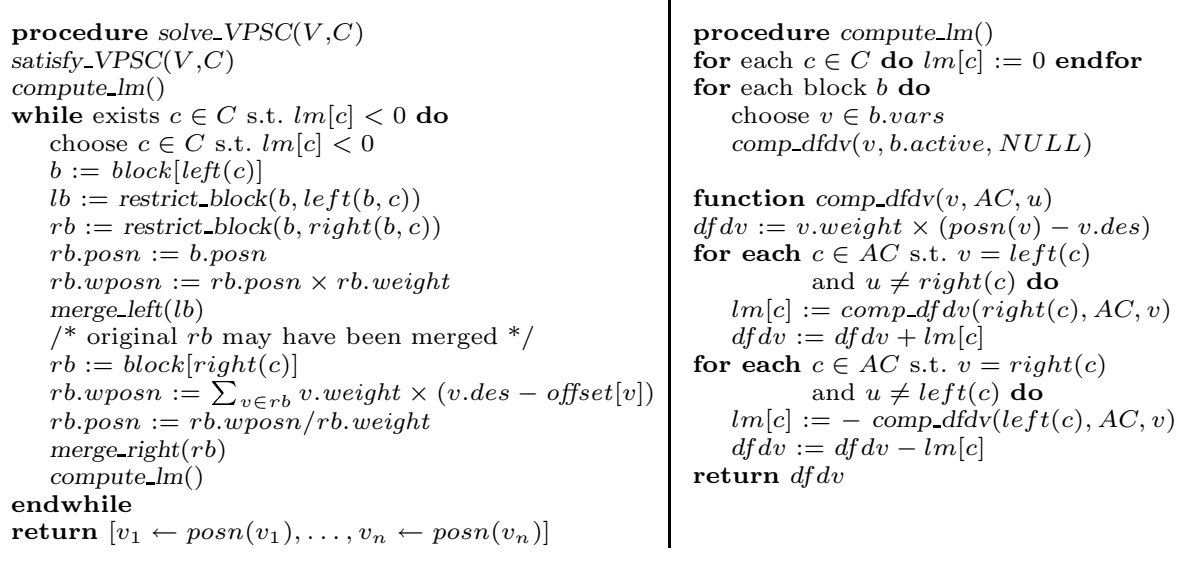

Fig. 3. Algorithm to find an optimal solution to a VPSC problem with variables $V$ and separation constraints $C$

Theorem 6. The procedure satisfy_VPSC $(V, C)$ has worst-case complexity $O(|V|+|C| \log |C|)$ with appropriate choice of priority queue data structure.

Since each block is placed at its optimal position one might hope that the solution returned by satisfy_VPSC is also optimal. This was true for the example above. Unfortunately, as the following example shows it is not always true.

Example 2. Consider the same blocks as in Example 1 but with the total order A,B,D,C. The algorithm works identically to the stage shown in Figure 2(b). But now we consider block $\mathrm{D}$, which overlaps with block $\mathrm{AB}$. We merge the blocks to create block ABD which is placed at 0.75, as shown in Figure 2(d). Now block $\mathrm{ABD}$ overlaps with block $\mathrm{C}$ so we merge the two to the final position 0.166 as shown in Figure 2(e). The result is not optimal.

The solution will be non-optimal if it can be improved by splitting a block. This may happen if a merge becomes "invalidated" by a later merge. It is relatively straight-forward to check if a solution is optimal by computing the Lagrange multiplier $\lambda_{c}$ for each constraint $c$. We must split a block at an active constraint $c$ if $\lambda_{c}$ is negative. Because of the simple nature of the separation constraints it is possible to compute $\lambda_{c}$ (more exactly $\lambda_{c} / 2$ ) for the active constraints in each block in linear time. We simply perform a depth-first traversal of the constraints in b.active summing v.weight $\times(\operatorname{posn}(v)-v . d e s)$ for the variables below this variable in the tree. The algorithm is detailed in Figure 3, It assumes the data structures in satisfy_VPSC and stores $\lambda_{c} / 2$ in the $\operatorname{lm}[c]$ for each $c \in C$. A full justification for this given in 13 .

Using this it is relatively simple to extend satisfy_VPSC so that it computes an optimal solution. The algorithm is given in Figure 3 , This uses satisfy_VPSC to find an initial solution to the separation constraints and calls compute_lm to compute the Lagrange multipliers. The main while loop checks if the current solution is optimal, i.e. if for all $c \in C, \lambda_{c} \geq 0$, and if so the algorithm 
terminates. Otherwise one of the constraints $c \in C$ with a negative Lagrange multiplier is chosen (we choose $c$ corresponding to $\min \left\{\lambda_{c} \mid \lambda_{c}<0, c \in C\right\}$ ) and the block $b$ containing $c$ is split into two new blocks, $l b$ and $r b$ populated by left $(b, c)$ and $\operatorname{right}(b, c)$ respectively. We define left $(b, c)$ to be the nodes in b.vars connected by a path of constraints from b.active $\backslash\{c\}$ to left $(c)$, i.e. the variables which are in the left sub-block of $b$ if $b$ is split by removing $c$. We define $\operatorname{right}(b, c)$ symmetrically. The split is done by calling the procedure restrict_block $(b, V)$ which takes a block $b$ and returns a new block restricted to the variables $V \subseteq$ b.vars. For space reasons we do not include the (straight-forward) code for this.

Now the new blocks $l b$ and $r b$ are placed in their new positions using the procedures merge_left and merge_right. First we place $l b$. Since $l m[c]<0, l b$ wishes to move left and $r b$ wishes to move right. We temporarily place $r b$ at the former position of $b$ and try and place $l b$ at its optimal position. If any of the "in" constraints are violated (since $l b$ wishes to move left the "out" constraints cannot be violated). We remedy this with a call to merge_left(lb). The placement of $r b$ is totally symmetric, although we must first allow for the possibility that $r b$ has been merged so we update it's reference to the (possibly new) container of $\operatorname{right}(c)$ and place it back at its desired position. The code for merge_right has not been included since it is symmetric to that of merge_left. We have also omitted references to the "out" constraint priority queues used by merge_right. These are managed in an identical fashion to "in" constraints.

Example 3. Consider the case of Example2. The result of satisfy_VPSC is shown in Figure 2(d). The Lagrange multipliers calculated for $c_{1}, c_{2}, c_{3}$ are 1.333, 2.333, and -0.333 respectively. We should split on constraint $c_{3}$. We break block ABCD into $\mathrm{ABC}$ and $\mathrm{D}$, and placing them at their optimal positions leads to positions shown in Figure 2(c). Since there is no overlap the algorithm terminates.

Theorem 7. Let $\theta$ be the assignment to the variables $V$ returned by solve_VPSC $(V, C)$. Then $\theta$ is an optimal solution to the VPSC Problem with variables $V$ and constraints $C$

Termination of solve_VPSC is a little more problematic. solve_VPSC is an example of an active-set approach to constrained optimization 15. In practice such methods are fast and lend themselves to incremental re-computation but unfortunately, they may have theoretical exponential worst case behavior and at least in theory may not terminate if the original problem contains constraints that are redundant in the sense that the set of equality constraints corresponding to the separation constraints $C$, namely $\{u+a=v \mid(u+a \leq v) \in C\}$, contains redundant constraints. Unfortunately, our algorithm for constraint generation may generate equality-redundant constraints. We could remove such redundant separation constraints in a pre-processing step by adding $\epsilon^{i}$ to the gap for the $i^{t h}$ separation constraint or else use a variant of lexico-graphic ordering to resolve which constraint to make active in the case of equal violation. We can then show that cycling cannot occur. In practice however we have never found a case of cycling and simply terminate the algorithm after a fixed maximum number of splits. 


\section{Results}

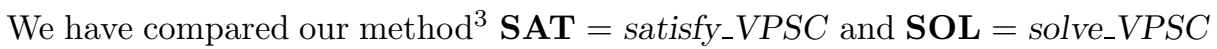
versus FSA, the improved Push-Force Scan algorithm [7] and QP quadratic programming optimization using the Mosek solver 16. For SAT, SOL and QP we compare with (_OO) and without orthogonal ordering constraints. We did not compare empirically with the Voronoi centering algorithm 10 since it gives very poor results, see Figure 4 .

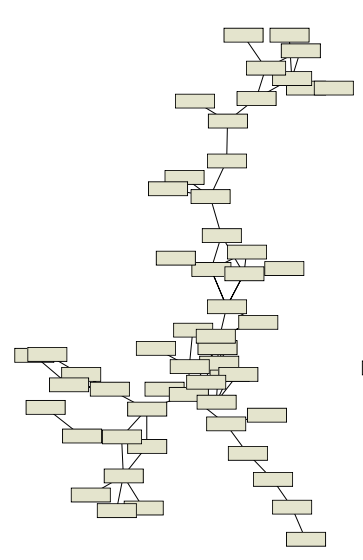

(a) Original layout

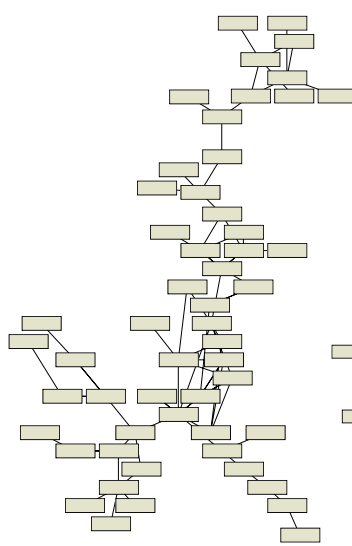

(d) SOL_OO=QP_OO

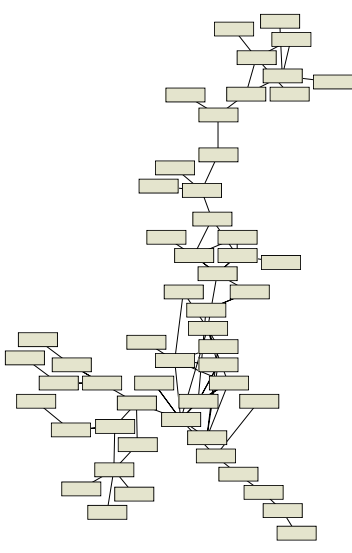

(b) SAT

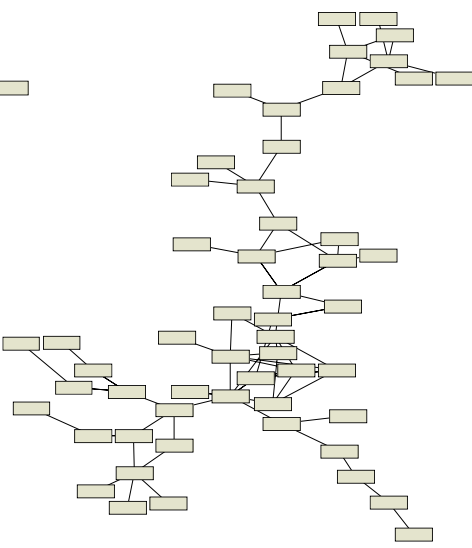

(e) FSA

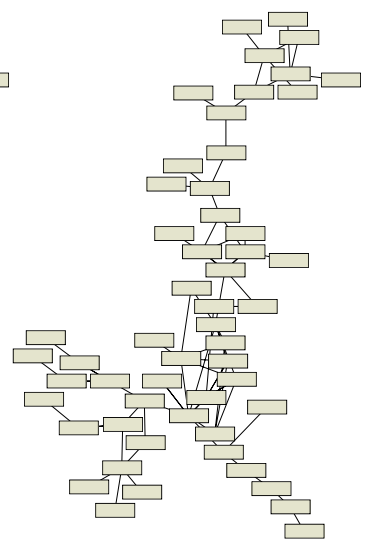

(c) $\mathrm{SOL}=\mathrm{QP}$

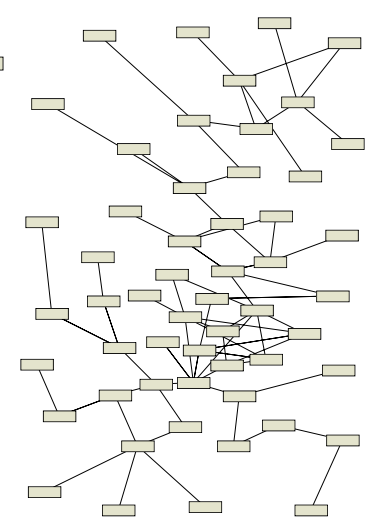

(f) Voronoi

Fig. 4. An example graph layout adjusted using various techniques

Figure 4 shows the initial layout and the results of the various node adjustment algorithms for a realistic example graph. There is little difference between the

${ }^{3} \mathrm{~A} \mathrm{C}++$ implementation of this algorithm is available from http://www.csse. monash.edu. au/ $\sim$ tdwyer. 


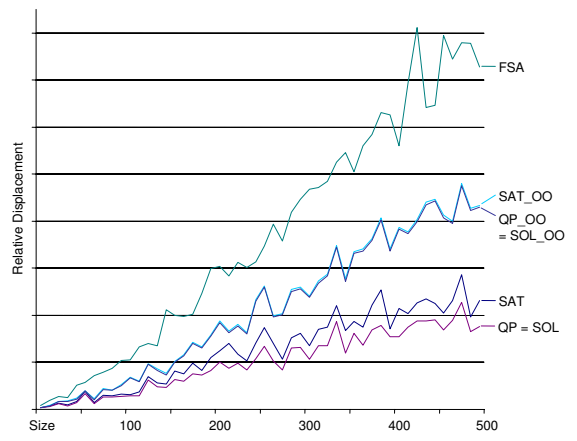

(a)

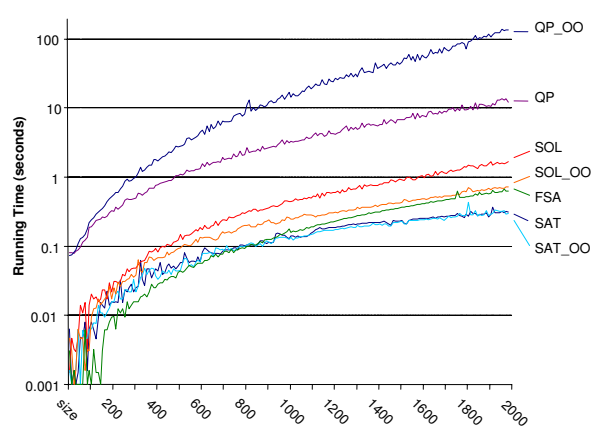

(b)

Fig. 5. Comparative (a) total displacement from original positions and (b) times

SAT and SOL results. We include a SOL result with the orthogonal ordering (SOL_OO) constraints which attacks the same problem as FSA. Clearly FSA produces much more spreadout layout. Lastly the Voronoi diagram approach loses most of the structure of the original layout.

Figure 5 gives running times and relative displacement from original position for the different methods on randomly generated sets of overlapping rectangles. We varied the number of rectangles generated but adjusted the size of the rectangles to keep $k$ (the average number of overlaps per rectangle) appoximately constant $(k \approx 10)$.

We can see that FSA produces the worst displacements, and that SAT produces very good displacements almost as good as the optimal produced by SOL and QP. We can see that SAT (with or without orthogonal ordering constraints) scales better than FSA. While both SOL and QP are significantly slower, SOL is an order of magnitude faster than $\mathrm{QP}$ in the range tested. Adding orthogonal ordering constraints seems to simplify the problem somewhat and SOL_OO requires less splitting than SOL while QP requires more processing time to handle extra constraints. Therefore SOL_OO is significantly faster than QP_OO and SAT_OO returns a solution very near to the optimal while remaining extremely fast. Overall these results show us that SAT is the fastest of all algorithms and gives very close to optimal results.

\section{References}

1. Battista, G.D., Eades, P., Tamassia, R., Tollis, I.G.: Graph Drawing: Algorithms for the Visualization of Graphs. Prentice Hall (1999)

2. Harel, D., Koren, Y.: Drawing graphs with non-uniform vertices. In: Proceedings of the Working Conference on Advanced Visual Interfaces (AVI'02), ACM Press (2002) 157-166

3. Friedrich, C., Schreiber, F.: Flexible layering in hierarchical drawings with nodes of arbitrary size. In: Proceedings of the 27th conference on Australasian computer science (ACSC2004). Volume 26., Australian Computer Society (2004) 369-376 
4. Marriott, K., Moulder, P., Hope, L., Twardy, C.: Layout of bayesian networks. In: Twenty-Eighth Australasian Computer Science Conference (ACSC2005). Volume 38 of CRPIT., Australian Computer Society (2005) 97-106

5. Misue, K., Eades, P., Lai, W., Sugiyama, K.: Layout adjustment and the mental map. Journal of Visual Languages and Computing 6 (1995) 183-210

6. Marriott, K., Stuckey, P., Tam, V., He, W.: Removing node overlapping in graph layout using constrained optimization. Constraints 8 (2003) 143-171

7. Hayashi, K., Inoue, M., Masuzawa, T., Fujiwara, H.: A layout adjustment problem for disjoint rectangles preserving orthogonal order. In: GD '98: Proceedings of the 6th International Symposium on Graph Drawing, London, UK, Springer-Verlag (1998) 183-197

8. Lai, W., Eades, P.: Removing edge-node intersections in drawings of graphs. Inf. Process. Lett. 81 (2002) 105-110

9. Gansner, E.R., North, S.C.: Improved force-directed layouts. In: GD '98: Proceedings of the 6th International Symposium on Graph Drawing, London, UK, Springer-Verlag (1998) 364-373

10. Lyons, K.A.: Cluster busting in anchored graph drawing. In: CASCON '92: Proceedings of the 1992 conference of the Centre for Advanced Studies on Collaborative research, IBM Press (1992) 327-337

11. Li, W., Eades, P., Nikolov, N.: Using spring algorithms to remove node overlapping. In: Proceedings of the Asia-Pacific Symposium on Information Visualisation (APVIS2005). Volume 45 of CRPIT., Australian Computer Society (2005) 131-140

12. Preparata, F.P., Shamos, M.I. In: Computational Geometry. Springer (1985) 359365

13. Dwyer, T., Marriott, K., Stuckey, P.J.: Fast node overlap removal. Technical Report 2005/173, Monash University, School of Computer Science and Software Engineering (2005) Available from www.csse.monash.edu.au/ tdwyer.

14. Weiss, M.A.: Data Structures and Algorithm Analysis in Java. Addison Wesley Longman (1999)

15. Fletcher, R.: Practical Methods of Optimization. Chichester: John Wiley \& Sons, Inc. (1987)

16. ApS, M.: (Mosek optimisation toolkit v3.2) www.mosek.com. 